\title{
Defining the appropriate waiting time between multiple-breath nitrogen washout measurements
}

\author{
To the Editor:
}

Static lung volume measurements allow objective assessment of total lung capacity (TLC), functional residual capacity (FRC) and residual volume, and assist in the diagnosis and management of lung disease $[1,2]$. The use of gas dilution techniques, such as multiple-breath nitrogen washout (MBNW), require minimal patient cooperation and allow FRC to be determined in those individuals unable to complete plethysmographic measurements. The MBNW technique is used clinically and for research, and provides accurate and repeatable measures in both children and adults $[3,4]$.

The 2005 American Thoracic Society (ATS) and European Respiratory Society (ERS) guidelines recommend waiting between repeated $\mathrm{MBNW}$ tests for $\geqslant 15 \mathrm{~min}$ and that in obstructed patients a period of $>1$ h may be required [2].

This study aimed to develop an evidence-based recommendation for the wait required between repeated MBNW tests using contemporary equipment and standardised collection protocols.

Children were tested in the Respiratory Laboratory of Princess Margaret Hospital for Children and the adults at the Respiratory Laboratory of Royal Perth Hospital, both located in Perth, Western Australia. The study was approved by the relevant ethics committees (EC LR.2008/127 and EC06-91), and written informed consent obtained from all participants and/or their parents, as relevant.

The study commenced in children between November 2006 and January 2008, and then expanded to the adult population from December 2007 to November 2009. Spirometry was only obtained in participants with lung disease according to current guidelines [5]. All MBNW tests were conducted according to the 2005 ATS/ERS criteria for the MBNW technique [2].

Children attended on a single occasion and were classed as healthy or as having lung disease, including asthma and cystic fibrosis. The initial MBNW (VMax Encore 229; Carefusion, Sydney, Australia) measurement of FRC was obtained for each child $(t=0)$. Children repeated the FRC measurement after waiting for $5 \mathrm{~min}$ then after waiting a further $15 \mathrm{~min}$, or after waiting for $15 \mathrm{~min}$ then $5 \mathrm{~min}$, with the testing order randomised.

The adults were classed as healthy, or as having obstructive or restrictive lung disease. On review of the paediatric data and considering that the time to clear gas from the lungs is dependent on disease severity, the protocol for adults was modified such that the time between MBNW tests was determined as a multiple of the initial washout time. An initial MBNW (Medgraphics Corporation, St Paul, MN, USA) was performed $(t=0)$ to provide the baseline washout time. The measurement was then repeated after waiting once, twice and three times their initial washout time, in randomised order.

Data (mean \pm SD) were normally distributed. Lung function is presented as standardised residuals derived from relevant prediction equations [6-9]. We defined acceptable between-test repeatability as a change in FRC $<10 \%$, in line with current MBW testing guidelines [10]. Power analysis showed that group sample sizes of 14 would detect a change of $10 \%$ assuming a SD of 0.35 (coefficient of variation, 18.75\%). Data were analysed using paired t-tests and a random-effects longitudinal regression model to assess the change in FRC from baseline.

Acceptable and repeatable FRCs were obtained in 19 healthy children and 18 with lung disease (eight with asthma and 10 with cystic fibrosis) aged $7-18$ years (mean \pm sD $12.76 \pm 3.18$ years). Spirometry (in standardised residuals) in the children with lung disease was: forced expiratory volume in $1 \mathrm{~s}\left(\mathrm{FEV}_{1}\right)$ $-1.48 \pm 1.75$; forced vital capacity (FVC) $-0.89 \pm 1.13$; and FEV $1 / F V C-1.21 \pm 1.51$. No clinically $(\geqslant 10 \%)$ or statistically significant differences were detected between baseline FRC (FRC0) and after 5 min (FRC5) or $15 \mathrm{~min}$ (FRC15) in either group (table 1). This was confirmed using random-effects regression modelling, with no associations between differences in FRC and waiting time, test order or disease status.

Measurements of FRC were obtained in 24 healthy adults (aged $35 \pm 16.3$ years), 16 adults with interstitial lung disease (aged 64.8 \pm 8.6 years) and 18 adults with chronic obstructive pulmonary disease (aged $61.6 \pm 17.1$ years)). Among those with restrictive lung disease, spirometry was: FEV1, $-1.66 \pm 1.22$; FVC, 


\begin{tabular}{|c|c|c|c|c|c|c|}
\hline & Children n & FRCo SR & FRCo L & $\Delta \mathrm{FRC}_{5} \mathrm{~L}$ & $\Delta \mathrm{FRC}_{15} \mathrm{~L}$ & \\
\hline Healthy & 19 & $-0.65 \pm 0.77$ & $1.90 \pm 0.57$ & $0.09 \pm 0.22$ & $0.01 \pm 0.15$ & \\
\hline \multirow[t]{2}{*}{ Lung disease } & 18 & $-0.47 \pm 1.64$ & $1.54 \pm 0.45$ & $0.01 \pm 0.13$ & $0.03 \pm 0.11$ & \\
\hline & Adults $\mathrm{n}$ & FRCo SR & FRCo L & $\Delta \mathrm{FRC}_{1 \times \mathrm{L}}$ & $\Delta \mathrm{FRC}_{2 \times \mathrm{L}}$ & $\Delta \mathrm{FRC}_{3 \times} \mathrm{L}$ \\
\hline $\begin{array}{l}\text { Healthy } \\
\text { Lung disease }\end{array}$ & 24 & $-0.66 \pm 1.44$ & $2.90 \pm 0.76$ & $-0.08 \pm 0.22$ & $-0.03 \pm 0.44$ & $0.03 \pm 0.31$ \\
\hline Restrictive & 16 & $-2.02 \pm 0.62$ & $1.99 \pm 0.42$ & $-0.07 \pm 0.20$ & $-0.11 \pm 0.27$ & $-0.03 \pm 0.19$ \\
\hline Obstructive & 18 & $1.56 \pm 2.23$ & $3.69 \pm 1.03$ & $-0.36 \pm 0.44^{* * *}$ & $0.01 \pm 0.29$ & $-0.15 \pm 0.46$ \\
\hline \multicolumn{7}{|c|}{$\begin{array}{l}\text { Data are presented as mean } \pm \text { SD unless otherwise stated. FRC } 0 \text { : baseline } F R C ; \text { SR: standardised residual; } \\
\Delta: \text { difference from FRC } 0 \text { in; FRC5: FRC measured after waiting } 5 \text { min; FRC } 15 \text { : FRC measured after waiting } \\
15 \text { min; FRC } 1 \times \text { : FRC measured after waiting for the initial washout time; FRC } 2 x: \text { FRC measured after } \\
\text { waiting for twice the washout time; FRC } 3 \times \text { : FRC measured after waiting for three times the initial washout } \\
\text { time. }{ }^{* * *}: p<0.001 \text {. }\end{array}$} \\
\hline
\end{tabular}

$-1.90 \pm 1.23$; and FEV1/FVC $0.41 \pm 1.28$. Among those with obstructive lung disease, spirometry was: FEV1 $-3.17 \pm 1.28$; FVC $-1.29 \pm 1.16$; and FEV $1 / F V C-3.30 \pm 1.36$. Waiting for the initial washout time had no effect on FRC in the healthy or restrictive lung disease groups. The obstructive lung disease group exhibited a clinically significant fall in FRC of $360 \mathrm{~mL}(-10.2 \%$; $<<0.001)$ after waiting for the washout time (table 1). This effect was not evident with longer waiting times (table 1). Random-effects regression analysis indicated that obstructive disease severity (assessed by FEV1/FVC) had no effect $(p=0.98)$ on the change in FRC between measurements. Similarly, restrictive disease severity (assessed by predicted TLC) also had no effect $(\mathrm{p}=0.69)$ on FRC.

This study investigated the time required for nitrogen levels in the lungs to return to baseline following repeated MBNW testing, thereby avoiding the introduction of measurement errors. To increase the generalisability of our findings, both children and adults were included, with varying degrees of lung disease severity.

In children we found that waiting $5 \mathrm{~min}$ (an average of 2.6 times the initial washout time) between measurements allowed nitrogen to return to baseline. In healthy adults and in adults with restrictive lung disease, a wait time of a single washout was sufficient for nitrogen to return to baseline. In adults with obstructive lung disease, a period of twice their initial washout time was required to measure FRC with acceptable repeatability. Nonetheless, waiting twice the initial washout time was, on average, $5.5 \mathrm{~min}$, and significantly shorter than the 15-60 min recommendation in the 2005 ATS/ERS guidelines. Previous infant MBNW testing guidelines [11] and the 2013 ATS/ERS MBNW consensus statement [10] recommend that a wait time of at least twice the washout period be used, and these data support that recommendation.

The study does have limitations. The protocol differed between the adult and paediatric groups, with the adult protocol informed by the paediatric data. We do not believe this alters our recommendations, as the children had a mean wait time of 2.6 times the initial washout time, which is in line with our recommendation of waiting at least twice the initial washout time in adults. This is further supported by the fact that the adults had more severe obstruction and, therefore, the likelihood of twice the washout time not being adequate in children is low. It should be acknowledged that patients with more severe lung disease that those in this study may require longer wait times and the observation of alveolar nitrogen levels prior to testing commencing, as recently recommended [10], would increase certainty around test commencement. We were not able to measure lung clearance index (LCI). However, errors that affect FRC will also impact LCI and we suggest that studies using ventilation distribution outcomes incorporate these findings into the measurement protocols.

In conclusion, we recommend a uniform approach to waiting time between MBNW tests, and to wait at least twice the initial washout time and to monitor post-test nitrogen levels before repeating the MBNW measurement. Further studies should examine further optimising these recommendations for all MBW outcomes. We believe our recommendation should inform lung function testing practices in paediatric and adult settings and will help optimise the quality of lung volume measurements. 
@ERSpublications

Repeated multiple-breath washout tests should be twice the washout time apart to reduce avoidable measurement errors http://ow.ly/HPgW4

Elizabeth R. Salamon ${ }^{1}$, Kevin R. Gain ${ }^{1}$ and Graham L. Hall ${ }^{2,3}$

${ }^{1}$ Respiratory Medicine, Royal Perth Hospital, Perth, Australia. ${ }^{2}$ Respiratory Medicine, Princess Margaret Hospital for Children, Perth, Australia. ${ }^{3}$ Telethon Kids Institute, University of Western Australia, Perth, Australia.

Correspondence: Graham Hall, Paediatric Respiratory Physiology, Telethon Kids Institute, PO Box 855, West Perth, Western Australia 6872, Australia. E-mail: graham.hall@telethonkids.org.au

Received: Sept 022014 | Accepted after revision: Dec 242014 | First published online: Feb 052015

Support statement: G.L. Hall is funded by an Australian National Health and Medical Research Council Fellowship (grant APP1025550). Funding information for this article has been deposited with FundRef.

Conflict of interest: None declared.

Acknowledgements: This work was presented at the 2010 European Respiratory Society Annual Congress in Barcelona, Spain, as an oral presentation. The authors would like to acknowledge the participants of this study from Princess Margaret Hospital and Royal Perth Hospital (Perth, Australia). They would also like to acknowledge statistician Michael Phillips from the Medical Research Foundation at Royal Perth Hospital for providing statistical analysis.

\section{References}

1 Pellegrino R, Viegi G, Brusasco V, et al. Interpretative strategies for lung function tests. Eur Respir J 2005; 26 : 948-968.

2 Wanger J, Clausen JL, Coates A, et al. Standardisation of the measurement of lung volumes. Eur Respir J 2005; 26 : 511-522.

3 Blonshine S, Foss C, Mottram C, et al. AARC clinical practice guideline: body plethysmography: 2001 revision and update. Respir Care 2001; 46: 506-513.

4 Newth CJ, Enright P, Johnson RL. Multiple-breath nitrogen washout techniques: including measurements with patients on ventilators. Eur Respir J 1997; 10: 2174-2185.

5 Miller MR, Hankinson J, Brusasco V, et al. Standardisation of spirometry. Eur Respir J 2005; 26: 319-338.

6 Hall GL, Thompson BR, Stanojevic S, et al. The Global Lung Initiative 2012 reference values reflect contemporary Australasian spirometry. Respirology 2012; 17: 1150-1151.

7 Quanjer PH, Stanojevic S, Cole TJ, et al. Multi-ethnic reference values for spirometry for the 3-95-yr age range: the Global Lung Function 2012 equations. Eur Respir J 2012; 40: 1324-1343.

8 Cook CD, Hamann JF. Relation of lung volumes to height in healthy persons between the ages of 5 and 38 years. J Pediatr 1961; 59: 710-714

9 Crapo RO, Morris AH, Clayton PD, et al. Lung volumes in healthy nonsmoking adults. Bull Eur Physiopathol Respir 1982; 18: 419-425.

10 Robinson PD, Latzin P, Verbanck S, et al. Consensus statement for inert gas washout measurement using multiple- and single-breath tests. Eur Respir J 2013; 41: 507-522.

11 Morris MG, Gustafsson P, Tepper R, et al. The bias flow nitrogen washout technique for measuring the functional residual capacity in infants. Eur Respir J 2001; 17: 529-536.

\section{Dual gas techniques for peripheral airway function: diffusing the issues}

To the Editor:

In the quest to investigate small airway function in a range of lung diseases, multiple-breath washout tests have been applied for their potential to represent the most peripheral air spaces $[1,2]$. The aim of reducing the burden on the patient has recently also revived interest in the single-breath washout. A single-breath washout test has now been proposed which involves an inhalation of $5 \% \mathrm{SF}_{6}, 26.3 \% \mathrm{He}, 21 \%$ $\mathrm{O}_{2}$ and the balance as $\mathrm{N}_{2}$ in order to obtain a so-called dual gas tracer (DTG) phase III slope [3]. After having been introduced as a practical and promising lung function tool [3] and as an early detection tool in cystic fibrosis lung disease [4], the DTG phase III slope is now advocated as a specific index of acinar function abnormality in children with mild asthma [5]. An editorial in the European Respiratory Journal [6], reflecting upon a DTG reproducibility study in normal subjects and chronic obstructive pulmonary 